\title{
Crack Characterization Using Image Analysis
}

James Grande

\section{GE Global Research Center, Niskayuna, NY 12309}

The microstructual characterization of thermal barrier coatings (TBC's) has been an important component in understanding the quality of the coatings and thus the ultimate performance in resisting metal substrate degradation in service. The focus of this paper is the characterization of cracks which may be a component of successful coating performance [1]. There are many ways to quantify crack morphology including orientation, actual length, number density, thickness, and overall horizontal and vertical length. However, a more complete characterization should include the determination of the degree to which a crack is perpendicular to the interface (vertical cracks).

A simple method for distinguishing directionality of identified cracks is to simply measure the aspect ratio or the angle of the longest feret. However, this characterization is limited in that crack morphology can be quite complicated with branching and angular directionality (Figure 1). A more robust methodology to characterizing cracks is to quantify not only the longest feret but to quantify the horizontal and vertical components of each crack. This involves adding up all of the horizontal and vertical intercepts for each crack and dividing by two. Some commercial packages have this measurement built-in, e.g., Clemex Inc.Vision image analysis software refers to this measurement as $\mathrm{X}$ and $\mathrm{Y}$ Projections, which makes the analysis quite straightforward.

The advantage of this technique is that it provides a more complete picture of the cracks and accounts for cracks that that may go off in various directions as well as ones that have significant branching (Figure 1). In addition, the $\mathrm{X}$ and $\mathrm{Y}$ projections can be used in conjunction with other shape parameters to ascertain the degree of branching and the tortuosity of the crack.

The processing steps involved require excellent metallographic preparation in order to minimize edge rounding and to image fine cracks that often emanate from larger ones. Once the image has been segmented to a binary image (Figure 2a), image processing can be performed in order to filter out pores (Figure 2b), connect smaller cracks to larger cracks which are in close proximity but not connected due to the 3-dimensional nature of cracks (Figure 2c), and finally thin the crack to approximate it's original thickness (Figure $2 \mathrm{~d}$ ) while retaining only $>100 \mu \mathrm{m}$ cracks.

Having different observers inspect cracks to consistently determine whether they are vertical or horizontal can be problematic if the cracks are not generally linear. Image analysis detection and quantification provides a non-subjective and robust method for crack assessment once processing and evaluation parameters are established. For example, when investigating the effects of large cracks (i.e., cracks longer than $100 \mu \mathrm{m}$ ) on mechanical properties, a general formula was developed after inspecting hundreds of large cracks that provided the most consistent and thorough representation of effective crack orientation. A Y projection to X projection ratio of 0.9 provided a good measure of vertical crack orientation and was used extensively in various coating evaluations. This crack ratio was also effective in accounting for cracks that would on first inspection be considered vertical but had sufficient horizontal branching to move the ratio below 0.9 and thus effectively be considered a horizontal crack. 
To quantify the level of crack branching, one can use the $\mathrm{X}$ projections with Feret measurements in order to generate a metric depending on the application and the morphology of interest.

Image analysis is an excellent tool which can characterize cracks in many different ways thus providing many avenues for determining correlates with various material properties. The use of intercepts can provide a more robust measure of crack morphology especially when used in conjunction with other more traditional measures.

\section{References}

[1] Friis, Martin, Persson, Christer, Wigren, Jan, Influence of particle in-flight characteristics on the microstructure of atmospheric plasma sprayed yttria stablilized $\mathrm{ZrO}_{2}$, Surface Coatings Technology 141 (2001) 115-127.

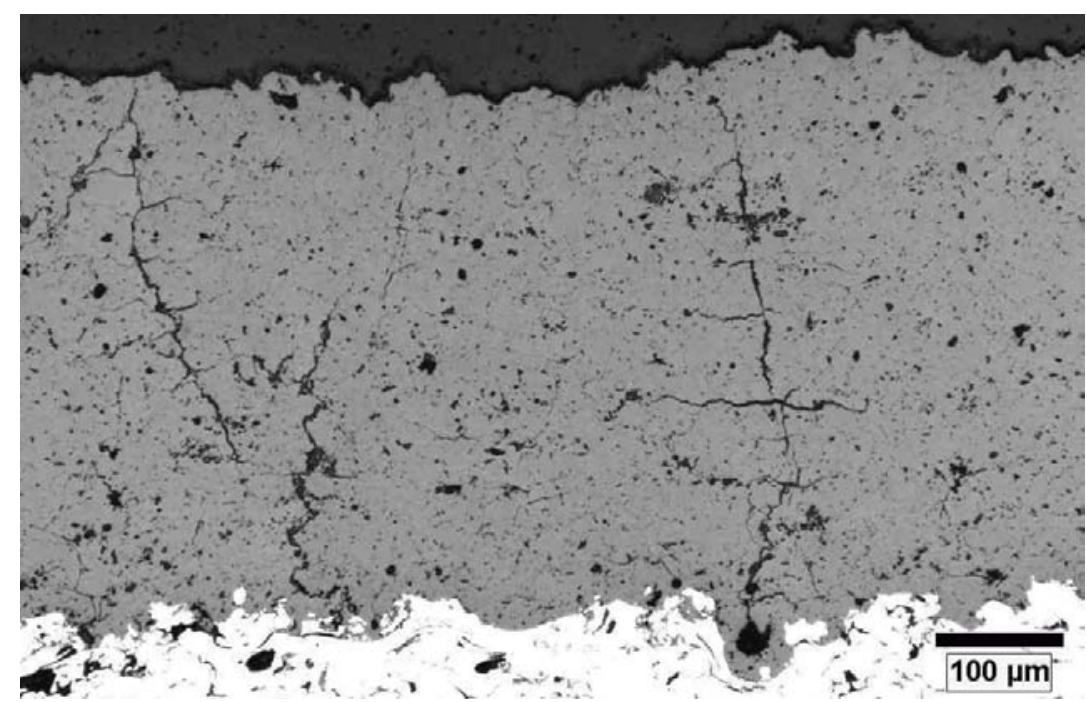

Figure 1.) TBC with various cracks depicting vertical cracks with branching and tortuosity.
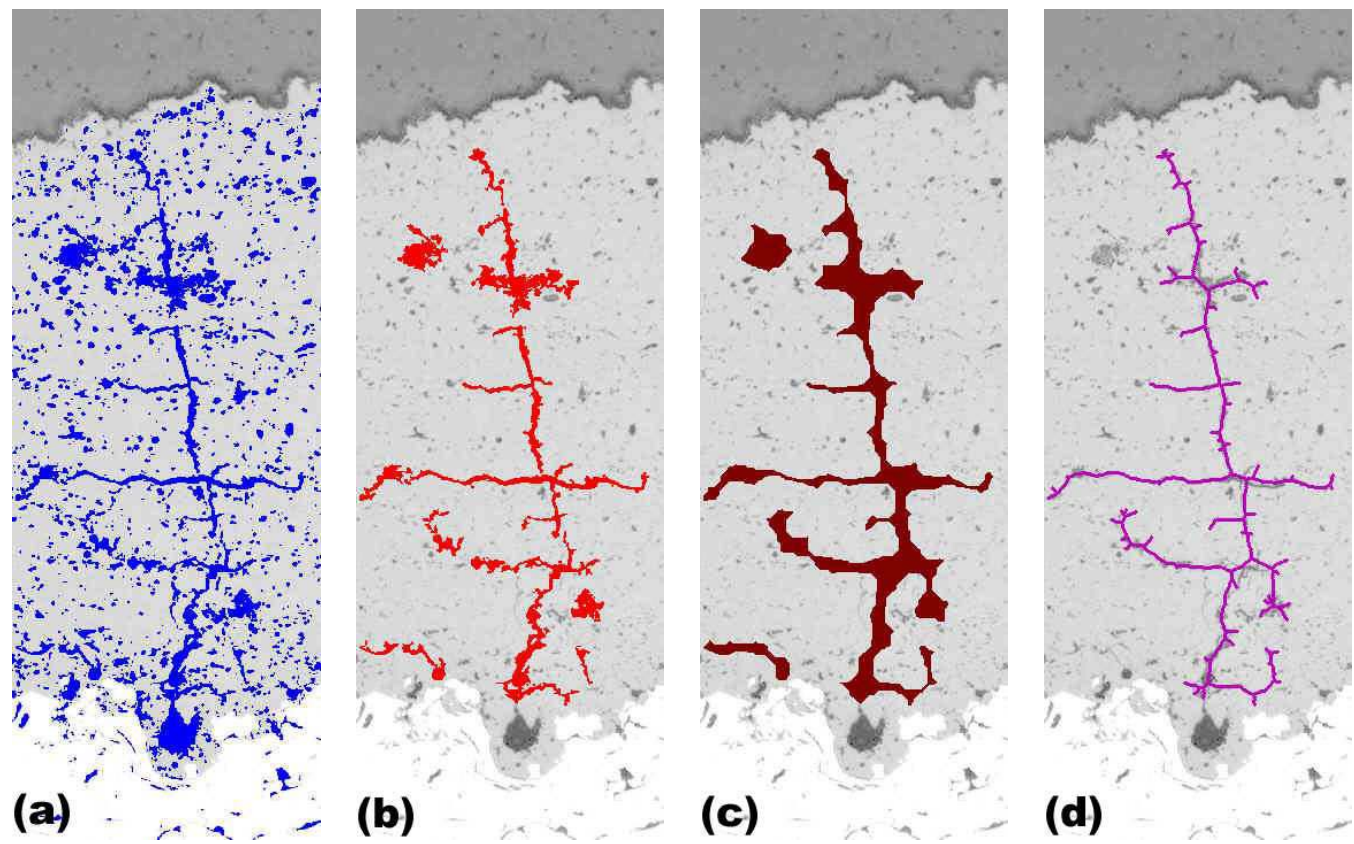

Figure 2.) Image processing steps for reconstructing large vertical cracks. 